\section{Cost-Effective Method to Synthesize Fluorescently Labeled DNA Size Stan- dards Using Cloned AFLP Fragments}

\author{
BioTechniques 34:1146-1148 (June 2003)
}

Fluorescent detection of PCR products is an established method in genome mapping, population genetics, and the individual identification of many organisms. The growing use of fluorescent detection and analysis techniques has facilitated high-throughput genotyping procedures, but running costs have been raised by the need to include a fluorescently labeled size marker for each sample. Recently, a method has been described for cost-effective labeling of size standards derived from a conserved region of plant chloroplast DNA (1). However, this technique requires as many as seven fluorescently labeled primers to generate 12 fragments of standard sizes. Here, we applied a different approach and propose a more cost-effective method to synthesize fluorescently labeled size standards. We are now constructing linkage maps of a herbaceous plant, Primula sieboldii, using AFLP (2), microsatellite markers, and fluorescently labeled size standards derived from $P$. sieboldii DNA.

Genomic DNA was extracted from a leaf of $P$. sieboldii with a DNeasy ${ }^{\circledR}$ Plant Mini Kit (Qiagen, Hilden, Germany). An AFLP ${ }^{\circledR}$ Core Reagent Kit (Invitrogen, Carlsbad, CA, USA) was then used for DNA digestion and adapter ligation, following the protocol provided by the manufacturer (except that we reduced the reaction volume to reduce costs). Briefly, genomic DNA (20-100 ng) was double-digested with EcoRI and $M s e \mathrm{I}$ in a $5-\mu \mathrm{L}$ reaction mixture. After inactivation of the restriction enzymes at $70^{\circ} \mathrm{C}$, adapters complementary to the EcoRI (E) and $\mathrm{MseI}(\mathrm{M})$ restriction sites were ligated. The ligation products were diluted 10fold and used as templates for preselective amplification, which was carried out by primers complementary to the adapter sequences with a $3^{\prime}$ extension of two nucleotides (E-AA and M-CA).
The amplification was performed in a $50-\mu \mathrm{L}$ reaction mixture containing 20 mM Tris-HCl, pH 8.4, 50 mM KCl, 0.2 $\mathrm{mM}$ each dNTP, $1.5 \mathrm{mM} \mathrm{MgCl} 2,1.5 \mathrm{U}$ Taq DNA polymerase, and $7.5 \mu \mathrm{L}$ diluted template DNA with the following thermal conditions: $3 \mathrm{~min}$ at $94^{\circ} \mathrm{C}, 30$ cycles of $30 \mathrm{~s}$ at $94^{\circ} \mathrm{C}, 30 \mathrm{~s}$ at $56^{\circ} \mathrm{C}, 30$ s at $72^{\circ} \mathrm{C}$, and finally $30 \mathrm{~min}$ at $72^{\circ} \mathrm{C}$. The amplification products created smeared banding patterns when separated on a $2 \%$ agarose gel. The products were cloned into $\mathrm{pCR}^{\circledR}-\mathrm{TOPO}^{\circledR}$ with a TOPO TA Cloning ${ }^{\circledR}$ Kit (Invitrogen). The presence/absence and the size of the inserts were analyzed by colony PCR, and appropriate clones were selected. Plasmid DNA was extracted from these clones, and the inserts were amplified by M13 pUC Sequencing Primers (Wako Pure Chemical Industries, Ltd., Osaka, Japan). The products were diluted 100-fold and used as templates for subsequent PCR. A TAMRA-labeled primer complementary to the EcoRI adapter sequence and a primer complementary to the MseI adapter sequence were used for fluorescent labeling of the DNA fragments. The labeling reactions for each fragment were performed separately in a $50-\mu \mathrm{L}$ reaction mixture comprising $1 \times$ PCR buffer $(10 \mathrm{mM}$ Tris-HCl, $\mathrm{pH}$ 8.3, $50 \mathrm{mM} \mathrm{KCl}, 100 \mathrm{mM}$ each dNTP, $0.02 \%$ Triton ${ }^{\circledR} \mathrm{X}-100$, and $0.01 \%$ gelatin), $1.5 \mathrm{mM} \mathrm{MgCl}_{2}, 1.25 \mathrm{U} \mathrm{Taq}$ DNA polymerase, $0.25 \mu \mathrm{M}$ each primer, and $5 \mu \mathrm{L}$ template DNA with the following conditions: $3 \mathrm{~min}$ at $94^{\circ} \mathrm{C}, 35$ cycles of $30 \mathrm{~s}$ at $94^{\circ} \mathrm{C}, 30 \mathrm{~s}$ at $56^{\circ} \mathrm{C}$, and $30 \mathrm{~s}$ at $72^{\circ} \mathrm{C}$, concluding with $10 \mathrm{~min}$ at $72^{\circ} \mathrm{C}$. The size of each labeled fragment was estimated by coelectrophoresis with a commercial set of size standards (GeneScan ${ }^{\circledR}$ TAMRA500; Applied Biosystems, Foster City, CA, USA) and least square sizing analysis by GeneScan software (Applied Biosystems). The resulting data were rounded to the nearest integers, which were used to define the size of the standard. The actual size of the fragments was not determined by sequencing because differences between actual and designated sizes do not matter for genotyping. Finally, equal volumes of each product were mixed, diluted to an appropriate fluorescent intensity, and used as standards. We randomly selected 34 recombinant clones, 14 of which were used as size standards, and Figure 1 shows an electropherogram of this set of standards.

To evaluate the reliability of the size standards, three microsatellites in a $P$. sieboldii plant (3) were amplified and electrophoretically separated with the developed size standard on $7 \%$ denaturing acrylamide gels (12 cm well-toread) in $0.5 \times$ TBE buffer using an ABI PRISM $^{\circledR} 377$ DNA sequencer equipped with GeneScan software. Data from three gels, each containing triplicate lanes from the same PCR amplification reaction were analyzed by the local Southern algorithm (4). Amplification of the three microsatellites yielded six fragments, resulting in a total of $54 \mathrm{ob}-$ servations. Standard deviations were calculated from estimated size differences within and between gels, ranging from 0.02 to 0.04 and from 0.03 to 0.05 nucleotides, respectively (Table 1). These results indicate the reliability of the synthesized size standard. The low standard deviation values reflect the fact that single alleles were analyzed and sized.

Cost analysis for routine production of the synthesized size standard showed that it is more economical, by a factor of about 128 , than a commercial size standard (Table 2). The costs for extracting DNA from the plant materi-

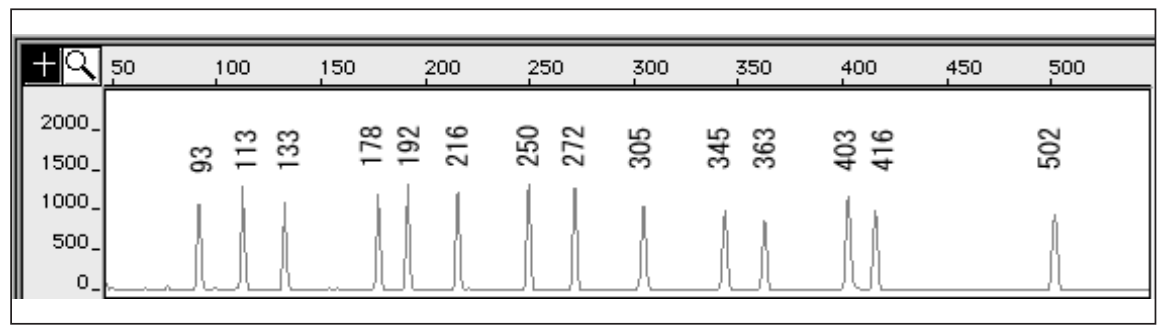

Figure 1. Electropherogram of the synthesized size standard. Sizes of the fragments (in base pairs) are indicated above each peak. 


\section{Benchmarks}

Table 1. Observed Differences in the Estimated Fragment Sizes for Microsatellites in $P$. sieboldii

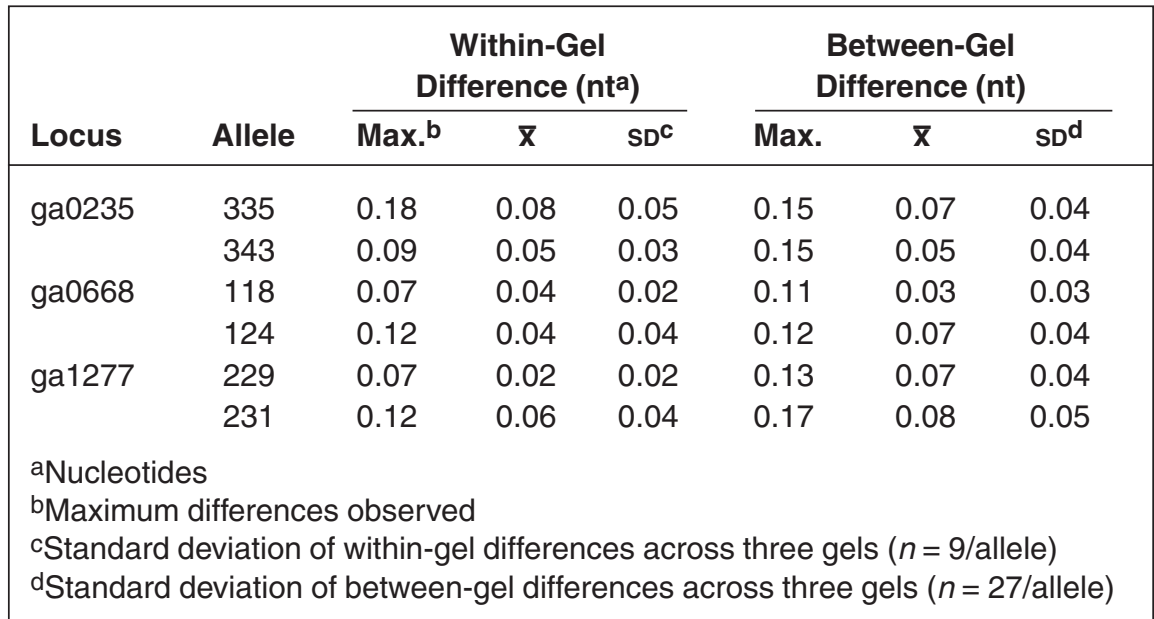

Table 2. Cost Analysis for Routine Production of the Synthesized Size Standard and Comparison with a Commercial Size Standard

\begin{tabular}{|lccccc} 
& \multicolumn{2}{c}{ Synthesized Size Standard } & & Commercial Size \\
\cline { 2 - 3 } & Expendables & Labor & Total & & Standard \\
\hline Unita cost & $\$ 9.30^{\mathrm{b}}$ & $\$ 13.60^{\mathrm{c}}$ & $\$ 22.90$ & \\
Cost/96 gel lanes & $\$ 0.17$ & $\$ 0.25$ & $\$ 0.42$ & $\$ 450$ \\
\hline
\end{tabular}

aOne unit for the synthesized size standard is defined as one synthesis of the 14 standard fragments by PCR with the fluorescently labeled primer in $50 \mu \mathrm{L}$ each reaction mixture, and one unit for the commercial size standard is defined as one package.

bOnly costs unique to the production of the standard (synthesis of the fluorescently labeled primer and PCRs) are included. Costs of extracting DNA from the plant material and amplified fragment library construction are excluded.

cAssuming $\$ 6.8 / \mathrm{h}$ for a laboratory technician and $2 \mathrm{~h}$ for one unit of the synthesized standard.

Note that the cost analysis is based on a 2003 exchange rate of 120 yen/dollar.

al and constructing the library of amplified fragments were estimated to be $\$ 140$, including all reagents and labor. However, the initial investment will be paid off because, once the fragment library is constructed, it can be routinely used to produce infinite amounts of size standards at low cost.

The biggest advantage of this method is that only one fluorescent primer is needed to label the sizing standard. Moreover, fragments of appropriate size are chosen from the recombinant clones so that many sets of sizing standards can be constructed, according to specific needs. Modification of this simple PCR approach to generate size standards may include the use of any recombinants of suitable size as template DNA. Another advantage of our method is that consis- tent nontemplated addition of adenine $(+\mathrm{A})$ to the fluorescently labeled strand by Taq DNA polymerase is expected, so batch-to-batch variations in the size of standard fragments due to variations in the degree of adenylation may be avoided. Since the $5^{\prime}$ end of the primer complementary to the MseI adapter ends with guanine, Taq DNA polymerase promotes additions of + A to the corresponding fluorescently labeled strand (5).

In summary, we have developed a cost-effective method to generate fluorescently labeled size standards using cloned AFLP fragments. This enabled us to amplify many fragments of different sizes using a single primer combination. Amplified fragments were cloned and several clones with appropriate size were selected. The inserts were amplified and used with a fluorescently labeled primer for the production of fluorescently labeled size standards by PCR. The protocol described here can be easily followed by laboratories equipped to perform standard molecular techniques and will substantially reduce costs of genotyping projects.

\section{REFERENCES}

1.Brondani, R.P. and D. Grattapaglia. 2001 Cost-effective method to synthesize a fluorescent internal DNA standard for automated fragment sizing. BioTechniques 31:793-800.

2.Vos, P., R. Hogers, M. Bleeker, M. Reijans, T. van de Lee, M. Hornes, A. Frijters, J. Pot, et al. 1995. AFLP: a new technique for DNA fingerprinting. Nucleic Acids Res. 23:4407-4414

3.Ueno, S., Y. Tsumura, and I. Washitani. Development of microsatellite markers in Primula sieboldii E. Morren, a threatened Japanese perennial herb. Conserv. Genet. (In Press.)

4.Southern, E.M. 1979. Measurement of DNA length by gel electrophoresis. Anal. Biochem. 100:319-323

5.Magnuson, V.L., D.S. Ally, S.J. Nylund, Z.E. Karanjawala, J.B. Rayman, J.I. Knapp, A.L. Lowe, S. Ghosh, and F.S. Collins. 1996. Substrate nucleotide-determined non-templated addition of adenine by Taq DNA polymerase: implications for PCR-based genotyping and cloning. BioTechniques 21:700-709.

This work was supported by Environmental Research and Technology Development Fund from Ministry of the Environment, Government of Japan. The authors would like to thank Mihoko Kawakami for providing samples of $\mathrm{P}$. sieboldii. Address correspondence to Dr. Saneyoshi Ueno, Genome Analysis Laboratory, Department of Forest Genetics, Forestry and Forest Products Research Institute, 1 Matsunosato, Tsukuba, Ibaraki, 305-8687, Japan. email:saueno@ffpri.affrc.go.jp

Received 2 December 2002; accepted 24 February 2003.

\section{Saneyoshi Ueno ${ }^{1,2}$, Yoshihiko}

Tsumura ${ }^{2}$, and Izumi

Washitani $^{3}$

${ }^{1}$ Japan Wildlife Research Center

Tokyo

${ }^{2}$ Forestry and Forest Products

Research Institute

Tsukuba

${ }^{3}$ The University of Tokyo

Tokyo, Japan 